\title{
ENC-2020-0196 \\ REDUCED ORDER MODELS APPLIED TO LAMINAR DIFFUSION FLAMES
}

\author{
Nicole Lopes M. B. Junqueira \\ Luís Fernando Figueira da Silva \\ Pontifícia Universidade Católica do Rio de Janeiro, Brazil \\ nicolelopes2@outlook.com; luisfer@puc-rio.br \\ Louise da Costa Ramos \\ ANSYS/France \\ louise.ramos@ansys.com
}

Abstract. Computational model of combustion chambers is a major topic of research on the past decades. The modelling of such physical systems enables the analysis of several flame properties, such as velocity, species mass fraction and temperature. However, there is a high computational burden associated with the parametric exploration and the complexity of such systems. To overcome this problem, reduced order models (ROM) have been used to predict the behavior of those systems, decreasing the cost associated to the computational modeling. Accordingly, this work presents modeling of non premixed laminar flames stabilized in the Gülder burner, using a skeletal kinetic mechanism. The computational fluid dynamics flame model has been developed using Fluent 2019 R3 for the isothermal and reactive cases, for different prescribed fuel inlet velocities. The analysis of the flow and flame structures are respectively by means of the axial and radial velocity components and the mass fraction of $\mathrm{CH}_{4}$ fields in the isothermal case; and the temperature, mass fraction of $\mathrm{OH}$ and $\mathrm{CO}$ for the reactive case. Then, the ROM is construct with StaticROM from Twin Builder based on the CFD results. The results of the ROM have a maximum absolute error of $0.0025 \mathrm{~m} / \mathrm{s}$ for the velocity ROM, 0.0737 for the mass fraction of $\mathrm{CH}_{4}, 98.91 \mathrm{~K}$ for the temperature, $1.4710^{-3}$ for the mass fraction of $\mathrm{OH}$ and 0.018 for the mass fraction of $C O$. These errors are acceptable for the isothermal case and for the temperature, however, for the mass fractions of $O H$ and $\mathrm{CO}$ it shows a significant difference since the concentration of these species is small.

Keywords: Computational Fluid Dynamics, Reduced Order Model, combustion, diffusion flames

\section{INTRODUCTION}

Combustion is a complex physical process, which involves an exothermic chemical reaction between fuel and oxidizer that releases energy in form of heat, and includes several coupled flow processes, such as mass and heat transfer, chemical kinetics, thermodynamics and fluid dynamics. Despite being one of the most important sources of energy in the world, this process produces pollutants that are harmful to health. Soot, for example, is associated with the increase incidence of respiratory and heart disease or carbon monoxide, that is toxic for humans and animals (Bourdrel et al., 2017; Turino, 1981). For this reason, several studies have been developed to further understand the combustion and avoid these nuisances (Wang, 2011).

The Gülder burner, which has a simple geometry, is often considered to represent industrial burners on the study of soot formation and oxidation processes (Escudero et al., 2016). Moreover, the diffusion flame stabilized in this burner produces a higher volumetric fraction of soot when compared with others burners. This burner has been used at the study of Jerez et al. (2019) where the formation of soot and polycyclic aromatic hydrocarbons is effected, and De Castro and Figueira da Silva (2019), where are presented measurements of temperature and the volumetric fraction of soot using, respectively, two-colours pyrometry and extinction method. Cruz et al. (2020) used three and two color pyrometry to measure soot temperature, also.

Computational models have been vastly applied to characterize and optimize the combustion processes and help on the control of the emission of pollutants, particularly soot and $\mathrm{NO}_{x}$ (Zhang et al., 2010; Cremer et al., 2002). The use of computational fluid dynamics (CFD) enables the analysis of several flame properties, such as temperature and the species mass fraction included on the chemical kinetic model (Celis and Figueira da Silva, 2015; Orbegoso et al., 2016). However, high-fidelity and complex models demand a significant cost due to the number of equations that needs to be 
solved simultaneously and the number of nodes on the mesh (Zhang et al., 2007).

Reduced order model (ROM) is a machine learning methodology which enables the possibility of reducing the burden of parametric exploration for complex systems, without compromising the characteristic behavior of the system (LeCun et al., 2015; Luboz et al., 2018). These models have been applied to different problems, such as thermal, mechanical and fluid dynamic systems (Asgari et al., 2017; Luboz et al., 2018; Xiao et al., 2015). Since the modelling of combustion demands a high cost, the application of reduced order models on these systems is the main motivation of this work.

Different approaches have been used to develop offline ROM, i.e, models that learn after the training data is collected (LeCun et al., 2015). In general, these techniques starts with a reduction method is applied to the data, for instance singular value decomposition (SVD) or proper order decomposition (POD), and then an interpolation/integration method is applied to learn the behavior of the system. Xiao et al. (2015) compared different methods of integration/interpolation, the Smolyak sparse grid and the second order Taylor series, using the POD approach for the Navier-Stokes equations of a flow passing a cylinder. Da Costa Ramos et al. (2020) applied the SVD to decompose the data coupled with the genetic aggregation response surface (GARS) to predict the behavior of a premixed inverted conical flame (Ben Salem et al., 2017).

The objective of this study is to construct and characterize a laminar methane/air diffusion flame stabilized on a Gülder burner, developed entirely using ANSYS software: Fluent for flow and combustion modeling, and Static ROM from Twin Builder, for the ROM. The ROM is applied on computational data obtained with Fluent for several diffusion flames, where the variable parameter is the prescribed fuel inlet velocity. This paper follows the methodology used by Da Costa Ramos et al. (2020) for the modeling of a premixed inverted conical flame.

The structure of this manuscript is as follows. First is presented the computational fluid dynamic model, describing the domain, mesh, chemical model and boundary conditions. Then, the procedure to create the ROM is shown. Finally, the results from CFD and ROM are presented and discussed for the isothermal and the reactive cases representative of laminar non-premixed flames, comparing those results in term of ROM error and computational cost.

\section{METHODOLOGY}

In this section, first the process of modeling the laminar diffusion flame with Fluent is presented, such as the definition of the domain, the mesh and the numerical simulation setup. Then, the steps to develop the reduced order model are outlined.

\subsection{Computational fluid dynamics}

Since the Gülder burner presents an axial symmetry and a laminar axisymmetric flame is modeled, the computational domain used is twodimensional, as shown in Fig.1 (Escudero et al., 2016; Jerez et al., 2019). The air enters through the annular region of the burner, which has a radius of $43.5 \mathrm{~mm}$. The fuel inlet tube length is $60 \mathrm{~mm}$ and the radius is $5.5 \mathrm{~mm}$. In this work, the domain is divided in two connected parts, as shown in Fig.1, one internal (part A) which includes the fuel inlet tube and the estimated mixture zone, where the chemical reactions are expected to occur. This region A is located above the fuel tube outlet, and has radius of $27 \mathrm{~mm}$ and a length of $47.5 \mathrm{~mm}$. The second, external, region (part B) contains the remaining of the domain, where only air and burned gas flow. Such a divided domain enables the use of different mesh sizes at each part, and eases the control of the number of computational nodes on the model, thus decreasing the associated computational cost.

In the domain shown in Fig. 1, the different boundary conditions are represented in different colours; yellow for axis, red for outlet, blue for inlet and grey for the walls. At the inlet boundaries the mole fraction, temperature and velocity are prescribed. Following (De Castro and Figueira da Silva, 2019), the fuel velocity $\left(v_{z \mid f, i n}\right)$ is constant for each simulation here developed, and will be specified later on this work. The air inlet velocity is constant, $v_{z \mid a, \text { in }}=0.6 \mathrm{~m} / \mathrm{s}$. Air and fuel have a specified temperature of $300 \mathrm{~K}$ and pressure of $1 \mathrm{~atm}$. The outlet is a pressure outlet boundary and all the walls are adiabatic and non catalytic. The burner walls, located between the inlets, have no-slip condition, whereas, the external boundary has the slip condition, which approximates a free boundary setup, as used in experiments. This slip lateral boundary condition has been adopted in order to ease the computation convergence.

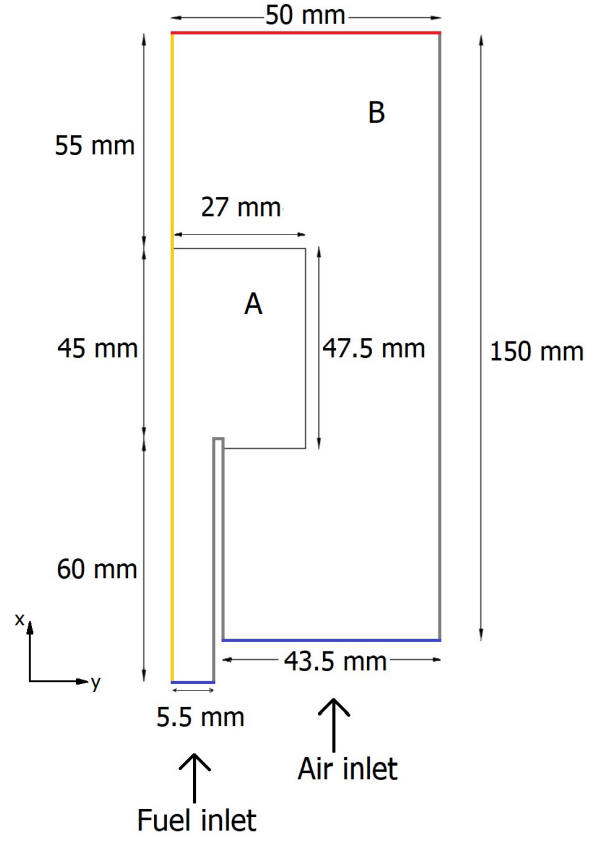

Figure 1: Representation of the computational domain and its dimensions. (A): internal part, (B): external part. 
However, this choice will be shown to lead to a curious flow structure at the exit of the fuel feed tube in the non-reactive cases.

The CFD models are developed using Fluent 2019 R3, which uses a finite volume method and a pressure based algorithm to solve the conservation equations for species mass, energy and momentum, and in this case, for multi-species methane/air combustion. Here the conservation equations are solved with the hypotheses of steady state, incompressible flow, ideal gas and the effects of gravity were not considered. The mass diffusivity, the kinetic-theory, and the Soret thermal diffusion effects are used to calculate the transport equations of the species in multi-component flow. The calculation of the fluid physical properties consider: incompressible ideal gas for density; ideal gas mixing law for thermal conductivity, viscosity and specific heat. In addition, the stiff chemistry solver has been used to integrate the reaction rate of the species transport equation. The solution methods for pressure-velocity coupling and spatial discretization of pressure are coupled and Presto, respectively. Concerning the spatial discretization of energy, species and momentum, the second-order upwind method is used.

In order to describe the chemical kinetics process with moderate computational time, a reduced kinetic model based on GRI-Mech 1.2 is used. This skeleton model, also called DRM19, is composed of 19-species and 83 reaction (Kazakov and Frenklach, 1984), and has been already used by Da Costa Ramos et al. (2020) for the modeling of inverted conical flames.

In this work, the internal part (A) of the domain has a characteristic mesh of size $10 \mu \mathrm{m}$ and $1 \mathrm{~mm}$ in the radial and axial directions, respectively, whereas the external part (B) has a mesh of size $1 \mathrm{~mm}$. These values have been chosen in order to focus the computational effort in the regions where flow and combustion properties vary significantly, for instance at the boundary layer at the burner walls and where the chemical reaction occurs. However, to adequately resolve the reactive region, a more refined mesh is necessary, which would increase the computational burden if used in the whole domain. For this reason, a dynamic mesh adaptation tool is used to refine the mesh where combustion occurs, using the temperature gradient with a refinement and coarsening criteria of $10 \mathrm{~K} / \mathrm{m}$ and $300 \mathrm{~K} / \mathrm{m}$, respectively. In other words, the cells with gradient values above the refinement threshold are refined, and the cells with gradient values below the specified value of coarse threshold are coarsened (ANSYS, 2009).

\subsection{Reduced order model}

Reduced order models is a methodology that enables the simple modelling of complex systems, without losing the most important characteristics of those (Luboz et al., 2018). It is a technique that has been used to reduce the burden linked to the parametric study of physical systems in the last decade (Asgari et al., 2017; Xiao et al., 2015). The Static ROM of Twin Builder is used as a model reduction technique. Its methodology is divided in four steps; (1) choosing the training dataset, (2) decomposing the data in simplified form, (3) retaining the main characteristics of the training set and (4) applying a machine learning interpolation, as depicted in Fig. 2.

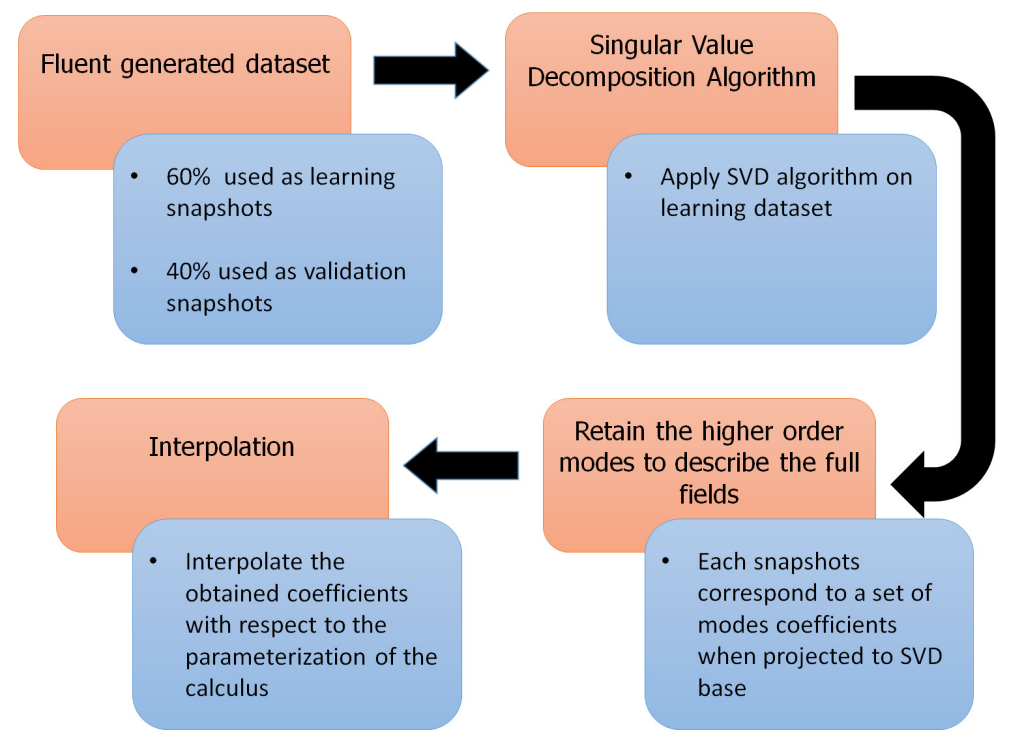

Figure 2: ROM construction scheme (Static ROM).

The first step consists on obtaining representative data of the system, of which $60 \%$ is used to learn the model and the remaining $40 \%$ to its validation. One may note that the learning set must include the extremes of the parameter space, in order to avoid an extrapolation when the created ROM is used to predict other states of the studied system. In the second step, a singular value decomposition (SVD) is used to decompose and obtain the system modes. The SVD organizes the 
modes in decreasing order, such that the higher mode values are those that retain the most important characteristics of the system of interest. At the third step, the decomposed system is filtered, retaining only the most important characteristics, i.e., the higher order modes. This procedure generates a reduced base of the learning data that describes the entire system.

Finally, the interpolation is performed with Genetic Aggregation Response Surface (GARS), which uses a selection process to determine which type of response surface (RS) better describes the system (Viana et al., 2017; Ben Salem et al., 2017). Since GARS can be defined as a weighted average of a set of response surfaces and has a cross-validation process, where the risk of an algorithm spreading the data set is estimated, this method presents an improved reliability when compared to classical response surface (Ben Salem et al., 2017). A model is then created to describe the behavior of the system for an arbitrary value of the variable parameter. Then, the prediction of the validation data may be performed.

The accuracy of the ROM is influenced by several factors, for instance the amount of data available, the number of modes used in the reduction of the learning set and the interpolation method error.

In this work, the construction of ROM is effected through the collection of computational fluid dynamic generated data, some of those are used to train the model and the remaining to validate it. Aiming the construction of reduced order models for each flow and flame properties.

\section{RESULTS AND DISCUSSION}

This section presents and analyzes the isothermal and reactive results of the laminar non premixed flame model obtained with Fluent and Static ROM.

\subsection{Learning and validation cases}

Six cases for the isothermal and reactive flowfields of the laminar non-premixed flame are modeled using CFD, where the prescribed fuel inlet velocity varies between $0.0175 \mathrm{~m} / \mathrm{s}$ and $0.0438 \mathrm{~m} / \mathrm{s}$, as shown at Tab. 1. Those results are then used to create the reduced order model of different properties. Concerning first the isothermal ROM three properties are studied; the axial and radial velocity components and the $\mathrm{CH}_{4}$ mass fraction. The reactive ROM involves; the temperature, the $\mathrm{OH}$ and $\mathrm{CO}$ mass fraction fields. For each ROM developed six datasets are available, one for each CFD case modeled, where four of those are used for learning the system and the remaining for validation. Table 1 shows the theoretical and the computed flame heights. The numerical flame height is obtained by determining the distance, along the computational domain centerline, between the tube exit and the position where the maximum value of the $\mathrm{H}$ atom mass fraction arises. The theoretical $L_{f, t h}$ value is computed using a relation between the flame height and the fuel volumetric flow rate proposed by McAllister et al. (2011). Although the theoretical height does not accurately describe flames with low Reynolds number, they exhibit the same trend; as the prescribed fuel inlet velocity increases, the flame height also increases. It may be expected that using a different scalar to define the flame height, such as $\mathrm{OH}$ or $\mathrm{O}$ mass fraction extreme, should lead to correspondingly larger values.

This section presents, first, the isothermal and the reactive CDF results, where the cases with the highest and lowest prescribed fuel inlet velocity are compared, i.e., cases 0 and 5 shown in Tab. 1. Then, an error analysis of the SVD reduction is performed, in order to determine the influence of the number of modes on the ROM accuracy. Finally, the results of reduced order model are given and analyzed. It should be emphasized that, all analysis performed in this work refer to the converged simulations results.

Table 1: Prescribed fuel inlet velocity $\left(v_{z \mid f, i n}\right)$, Reynolds number (Re), Froude number (Fr), theoretical and computed flame length $\left(L_{f}\right)$ for each case studied.

\begin{tabular}{|c|c|c|c|c|c|}
\hline Cases & $v_{z \mid f, \text { in }}[\mathrm{m} / \mathrm{s}]$ & $\mathrm{Re}$ & $\mathrm{Fr}$ & $L_{f, t h}[\mathrm{~cm}]$ & $L_{f, c}[\mathrm{~cm}]$ \\
\hline 0 & 0.0175 & 11.82 & 0.0533 & 2.26 & 1.42 \\
\hline 1 & 0.0211 & 14.26 & 0.0642 & 2.73 & 1.50 \\
\hline 2 & 0.0264 & 17.84 & 0.0803 & 3.42 & 1.90 \\
\hline 3 & 0.0317 & 21.43 & 0.0965 & 4.10 & 2.42 \\
\hline 4 & 0.0370 & 25.00 & 0.1126 & 4.79 & 2.83 \\
\hline 5 & 0.0438 & 29.6 & 0.1133 & 5.67 & 3.50 \\
\hline
\end{tabular}

\subsection{Isothermal flow results}

The isothermal behavior is initially studied to assess the laminar flow at the fuel tube outlet and to characterize the diffusion of $\mathrm{CH}_{4}$ throughout the domain. To represent the isothermal flow the axial and radial velocity components and the mass fraction of $\mathrm{CH}_{4}$ are used. 


\subsubsection{Computational fluid dynamics}

In order to discuss the influence of the prescribed fuel inlet velocity change, a qualitative comparison of the isothermal results is depicted in Fig. 3 for cases 0 and 5 (Tab. 1). The axial velocity component field, Fig. 3a, enables the analysis of the boundary layer development at the external burner wall and fuel fully developed flow at the fuel feed tube. Also, an unexpected region of re-circulation due to the detachment of the fuel flow is seen at the region of lowest velocity. This curious flow structure is an artifact related to the slip lateral wall boundary condition and to the low Reynolds number of the studied flows. This recirculation zone bears resemblance to classical boundary layer separation under adverse pressure gradient. Nevertheless, to a ROM application, the presence of this recirculation zone is considered to lead to a more challenging flow structure than that associated to pure longitudinal fluid motion. Comparing both cases, the region of re-circulation for the case 0 (left side of Fig. 3a) enters the fuel inlet tube more than the case with higher prescribed fuel inlet velocity (right side of Fig.3a). The radial velocity component is depicted at Fig. $3 \mathrm{~b}$ where it may be observed that in both cases a large part of the domain has a zero radial velocity component, which is explained by flow field continuity, under the hypotheses of steady-state, two-dimensional and incompressible fluid. Indeed, when the flow becomes developed, the velocity stops varying at the axial direction and the radial component of the velocity becomes constant. Two non-zero radial velocity component regions are noted near the fuel tube outlet, one negative and other positive, thereby indicating the mixture of fuel and air. Concerning the velocity field, the main difference between cases 0 and 5 is the radial velocity component, which absolute value at the fuel tube outlet is higher intensity at the latter case, as it could have been expected from the higher corresponding fuel flow velocity.

Figure $3 \mathrm{c}$ shows the mass fraction of $\mathrm{CH}_{4}$ for cases 0 and 5, where the outermost blue region contains only air, and the red region is solely methane. The white line represents the stoichiometric surface for the methane/air mixture, which is associated to a $\mathrm{CH}_{4}$ mass fraction of $\mathrm{Y}_{C_{4}, s t}=0.055$. The process of mixing the reagents occurs due to the mechanisms of mass transport through advection and diffusion. Concerning now case 0, Fig. 3c (left) one may verify that the stoichiometric surface is anchored at the fuel tube outlet and it extends in the axial direction until it approaches the axis of symmetry on the internal part of the domain, part A. In case 5, Fig. 3c (right), the stoichiometric surface also originates at the fuel tube outlet and only approaches the axis of symmetry on the external part of the domain, part B as shown at Fig. 1. This difference between the corresponding cases can be explained by the increase of the prescribed fuel inlet velocity.

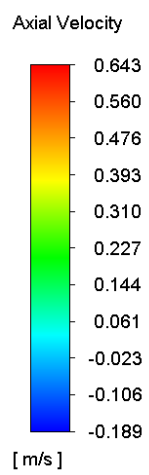

(a) Axial velocity component.

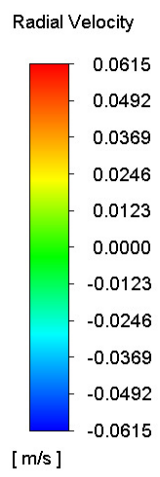

(b) Radial velocity component.

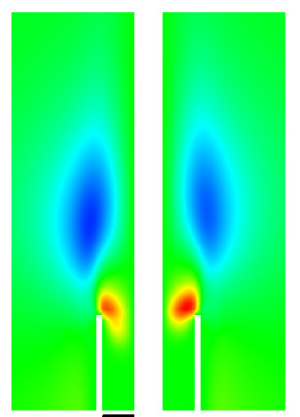

$5.5 \mathrm{~mm}$

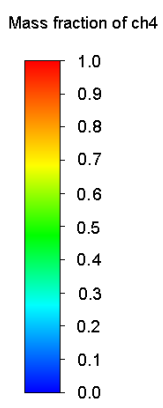

(c) Mass fraction of $\mathrm{CH}_{4}$.

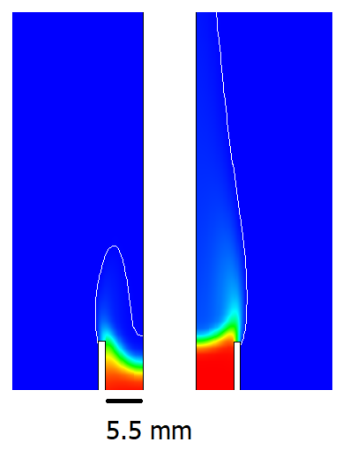

(left) and case 5 (right).

\subsubsection{Reduced order model}

Here, first the influence of the number of modes on the model accuracy for each reduced order model created is analyzed, followed by the comparison between the results obtained with CFD and ROM.

Four cases (cases 0, 2, 4, 5 at Tab.1) are used as learning data points for the construction of the isothermal ROM of the velocity components (axial and radial) and $\mathrm{CH}_{4}$ mass fraction. For model validation purposes, cases 1 and 3 of Tab. 1 are employed. Figure 4 compares the maximum absolute error resulting from the SVD reduction as a function the number of modes used in the construction of each ROM, where each colored line represents the variation of this error for each case. Figures $4 \mathrm{a}$ and $4 \mathrm{~b}$ show a similar behavior, i.e. for each considered case a high error results if only the first SVD mode is used. Indeed, for most cases, except for case 3, the maximum absolute error lies above $0.02 \mathrm{~m} / \mathrm{s}$ for the velocity ROM and is greater than 0.10 for the mass fraction of $\mathrm{CH}_{4}$, characterizing an underfitting problem. As the number of modes increases, the error decreases, achieving a 0 error when using the maximum number of modes, which does not occurs at validation cases, characterizing an overfitting problem. For this reason, three modes are used to create the isothermal reduced order model of velocity and mass fraction of $\mathrm{CH}_{4}$, thus leading to a maximum absolute error of $0.0025 \mathrm{~m} / \mathrm{s}$ and 
0.0737 , respectively.

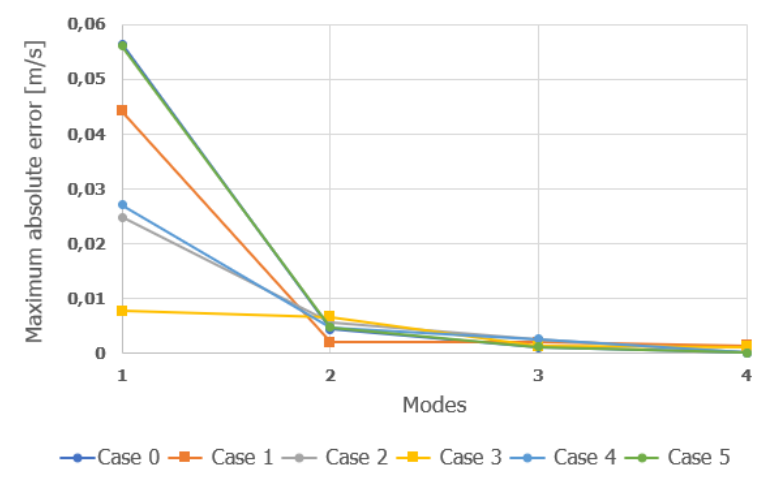

(a) Velocity.

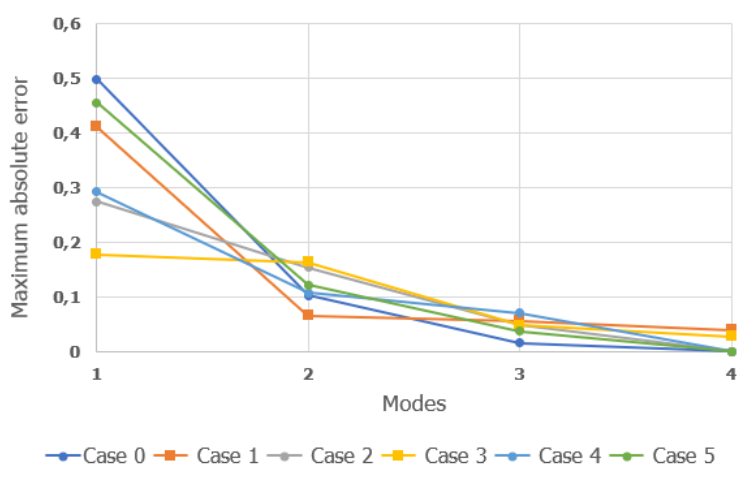

(b) Mass fraction of $\mathrm{CH}_{4}$.

Figure 4: Maximum SVD reduction absolute error $\times$ number of modes. Circle: learning data; Square: validation data.

Figure 5 exhibits a qualitative comparison of the results obtained with CFD and ROM for the axial velocity component and $\mathrm{CH}_{4}$ mass fraction, for case 3 from the validation set. For both properties, the ROM fields are qualitatively similar, without major differences when compared to the corresponding results calculated by CFD. The axial velocity component field, Fig. 5a, presents in both results the air and fuel boundary layers and the flow re-circulation. The mass fraction of $\mathrm{CH}_{4}$ field, Fig. 5b, shows the same outline for the stoichiometric surface (represented by the white line), but the ROM field shows a slightly larger surface than the CFD profile. In fact, as shown in the error analysis, an error of $0.0025 \mathrm{~m} / \mathrm{s}$ for the velocity and an error of 0.0737 for the mass fraction of $\mathrm{CH}_{4}$ have been obtained, which might be responsible for these small differences.

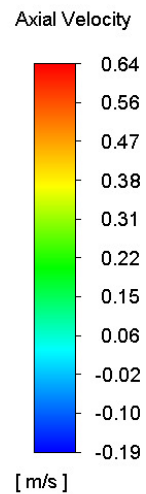

$[\mathrm{m} / \mathrm{s}]$

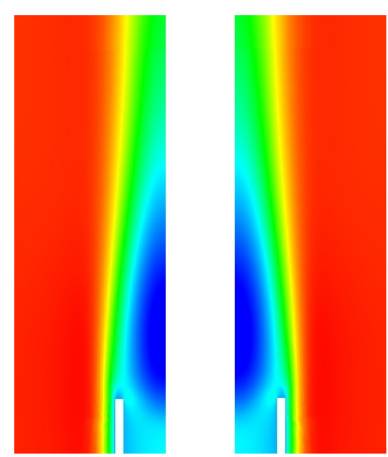

$5.5 \mathrm{~mm}$

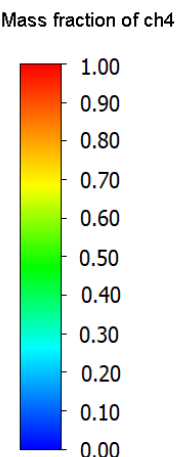

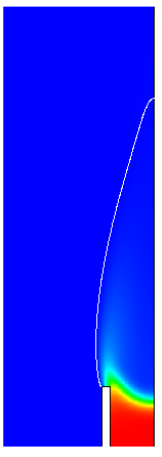

$5.5 \mathrm{~mm}$

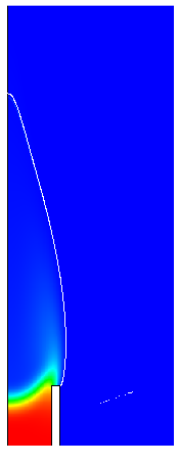

(b) Mass fraction of $\mathrm{CH}_{4}$.

(a) Axial velocity component

Figure 5: Comparison between isothermal flow results obtained with CFD (left) and ROM (right) for the validation case 3.

\subsection{Reactive flow results}

The outcome of the reactive flow simulations allows an analysis of several flame properties, such as species mass fraction, velocity and temperature. In order to describe the classical structure of the diffusion flame. Here, for the sake of brevity, only the temperature, the $\mathrm{OH}$ and $\mathrm{CO}$ mass fraction fields are analyzed.

\subsubsection{Computational fluid dynamics}

To underscore the influence of the Reynolds number of the fuel flow on the laminar diffusion flame structure, a comparative analysis is developed between cases 0 and 5 (given in Tab. 1). The temperature field depicted at Fig. 6a, shows that the region with the highest temperature characterizes the reactive and the burned gases, where, for case 0 the maximum temperature is $2138 \mathrm{~K}$ and for case 5 it is $2153 \mathrm{~K}$. One should note that the adiabatic flame temperature for methane/air is slightly higher $(2236 \mathrm{~K})$, which means the obtained results has about $4 \%$ of discrepancy from the expected adiabatic flame temperature. Also, along the axial direction of the flow, there is a decrease in temperature downstream the reactive and burned gases zone, characterizing the mixture of the combustion products, which are in high temperature, with 
the air, which has ambient temperature. It should be noted that, the case with the lowest Reynolds number is characterized by heat conduction into the fuel inlet tube, which is not observed in the case with the highest Reynolds number. This effect may be explained by the length of the flame; for small Reynolds number, the flame has a short length, such that the reactive region is close to the burner, thus heating the fresh gases which are inside and also causing species mass diffusion towards to the inlet tube region.

Figures $6 \mathrm{~b}$ and $6 \mathrm{c}$ present the mass fraction field of hydroxyl radical $(\mathrm{OH})$ and carbon monoxide $(\mathrm{CO})$, respectively. The mass fraction field of hydroxyl is mainly present at the flame sheet (McAllister et al., 2011), therefore, enables the identification of the shape and position of flame. Near the burner wall a higher concentration of $\mathrm{OH}$ may be found, since this region is where the flame is anchored. Further downstream, $\mathrm{OH}$ concentration decreases along the axial direction of the flow due to its dilution in the accumulated combustion products. Comparing the results of cases 0 and 5, a remarkable difference is the height of the flame, being higher for the case of higher Reynolds number, as can be seen in Tab. 1.

Concerning now, the carbon monoxide mass fraction field, Fig. 6c, this species is formed within the flame and its concentration gradually decreases downstream the flame sheet. It is important to note that also one relevant difference between the two cases is the length of the $\mathrm{CO}$ region, that is larger for case 5. Also, the CO mass fraction has a maximum concentration of 0.0526 , whereas for case 0 is 0.0514 . Another difference is that for case 0 , the mass fraction field of this substance exhibits a non negligible value within the fuel tube, which underscores the high temperature region, as shown at Fig. 6a. This phenomenon justifies the choice of including the fuel inlet tube in the modeling instead of starting the domain at the burner's outlet, since for small Reynolds numbers there is a high temperature region with the presence of burned gases inside the the fuel tube.

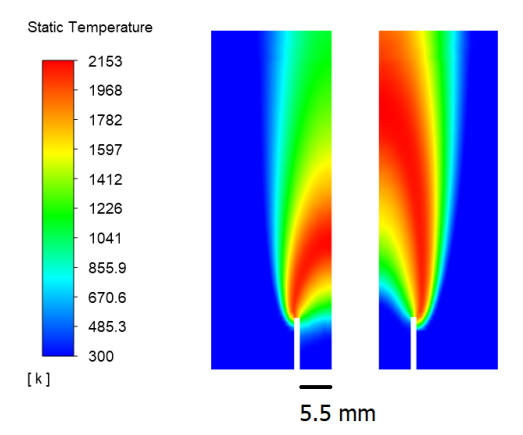

(a) Static temperature .

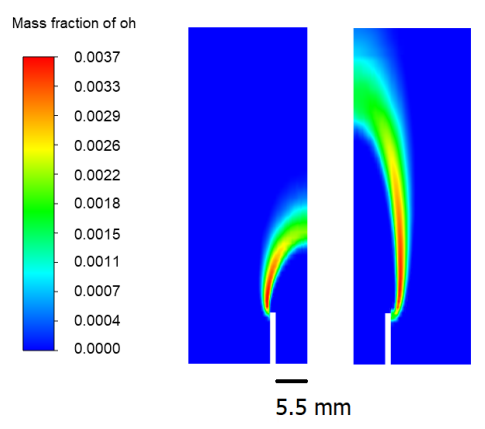

(b) Mass fraction of $\mathrm{OH}$.

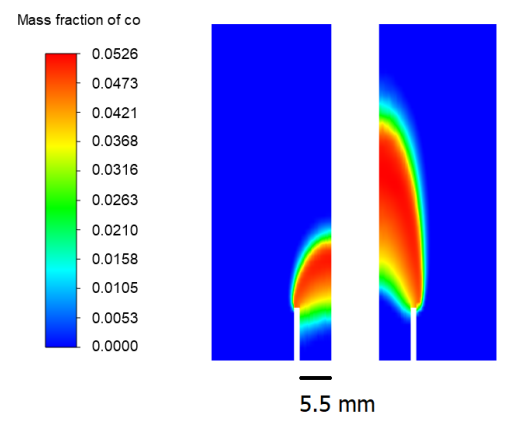

(c) Mass fraction of CO.

Figure 6: Comparison of reactive flow results obtained through CFD for case 0 (left) and case 5 (right).

\subsubsection{Reduced order model}

Before comparing the results of the reduced order model for the reactive case, an analysis of the influence of the number of modes on the reconstruction error is presented. Following the above-discussed isothermal results, for the construction of the reactive ROM of the static temperature, $\mathrm{CO}$ and $\mathrm{OH}$ mass fraction, cases $0,2,4$ and 5 of tab.1 are used as learning data points. Figure 7 shows the corresponding maximum absolute SVD reduction error for the temperature and $\mathrm{OH}$ mass fraction in function of the number of modes. The maximum absolute error for the mass fraction of $\mathrm{CO}$ is not shown, for the sake of brevity, because its behavior is quite similar to the results for the mass fraction of OH. Regarding the velocity components, the results obtained with CFD and ROM do not presents remarkable differences between them, having a maximum absolute error of $0.0019 \mathrm{~m} / \mathrm{s}$ with three modes, and are not plotted either.

Figures $7 \mathrm{a}$ and $7 \mathrm{~b}$ shows the same tendency as in the isothermal case, i.e., the error decreases with number of modes. For the temperature, with three modes the maximum absolute error for all cases are below $100 \mathrm{~K}$. It is interesting to remark that the experimental measurement of flame temperatures seldom exhibits an uncertainty inferior to $100 \mathrm{~K}$. Therefore, even with few learning cases the SVD reduction error is smaller than the experimental uncertainty (Cruz et al., 2020). It is noticed that for the validation cases in the $\mathrm{OH}$ mass fraction data, the SVD error does not decrease as fast as the other properties, such as, temperature and velocity in the isothermal case. The ROM's that has been developed for the static temperature, $\mathrm{CO}$ and $\mathrm{OH}$ mass fraction in this work, uses three modes, which leads to a maximum SVD reduction absolute error of $98,91 \mathrm{~K}, 0.018$ and $1.4710^{-3}$, respectively.

In order to illustrate the reconstruction effect on the reactive flow properties, Fig. 8 shows a qualitative analysis of the results obtained with $\mathrm{CFD}$ and ROM for temperature, mass fraction of $\mathrm{OH}$ ans $\mathrm{CO}$. The results in this figure allow to observe that the temperature fields are very similar, in particular, both results presents a heat conduction region into the fuel inlet tube and the temperature reaches its maximum in the region of the flame front. However, the values of the maximum temperature calculated by CFD is $2142 \mathrm{~K}$ and that predicted by ROM is $2068 \mathrm{~K}$, with an error of $74 \mathrm{~K}$ and a relative error of $1.87 \%$. 


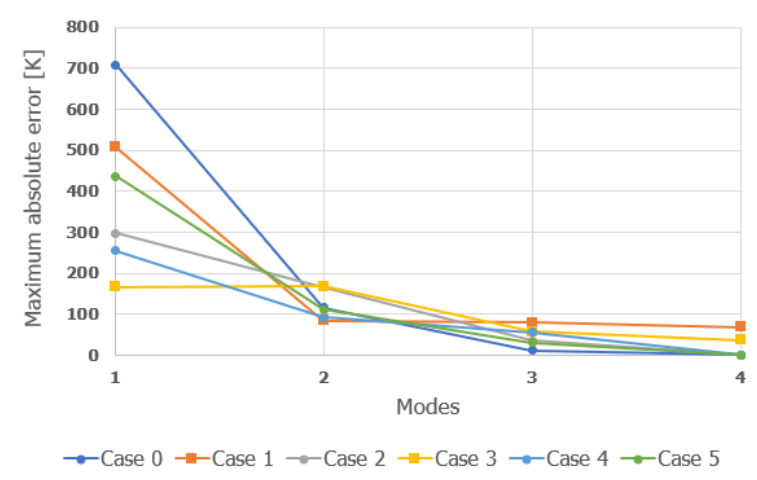

(a) Static temperature.

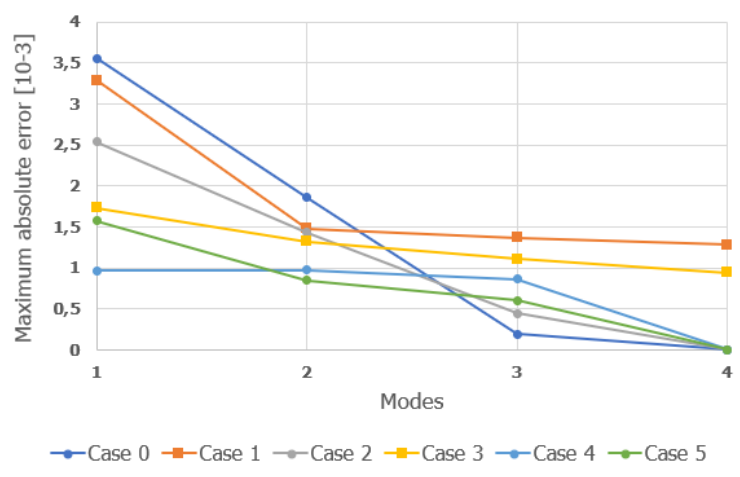

(b) Mass fraction of $\mathrm{OH}$

Figure 7: Maximum SVD reduction absolute error $\times$ number of modes. Circle: learning data; Square: validation data.

The mass fraction fields, on the other hand, show that for the $\mathrm{OH}$ mass fraction, Fig. $8 \mathrm{~b}$, the ROM is not able to predict the complete behavior along the flame front, with a relative error of $35.56 \%$. Indeed, the $\mathrm{OH}$ mass fraction field obtained through ROM is seen to be spread in the region near the symmetry axis. The results in this figure also, show that the region of high concentration does not extend as far downstream the tube exit as the CFD results.

Concerning now the mass fraction of $\mathrm{CO}$, Fig. 8c, there is also a significant qualitative discrepancy between the CFD and the ROM results, the relative error is $15.11 \%$ for case 1 . Indeed, the length and thickness of the region with the highest concentration of $\mathrm{CO}$ are higher in the result obtained by CFD than by ROM. Another significant point is that in the ROM result of the carbon monoxide, the diffusion of this chemical species is larger, causing a concentration of $\mathrm{CO}$ in regions where does not exist in CFD results, i.e. inside the fuel inlet tube and at the mixing region.

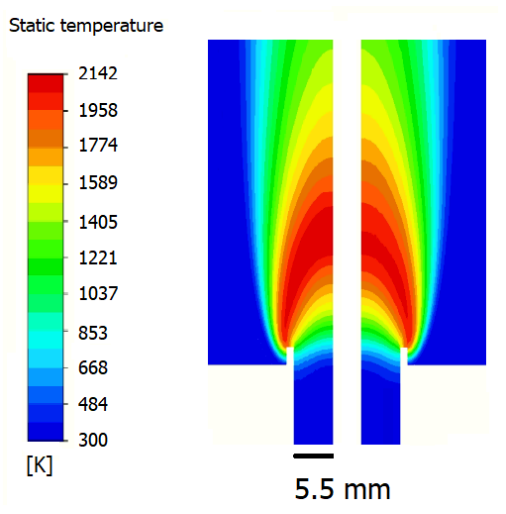

(a) Static temperature .

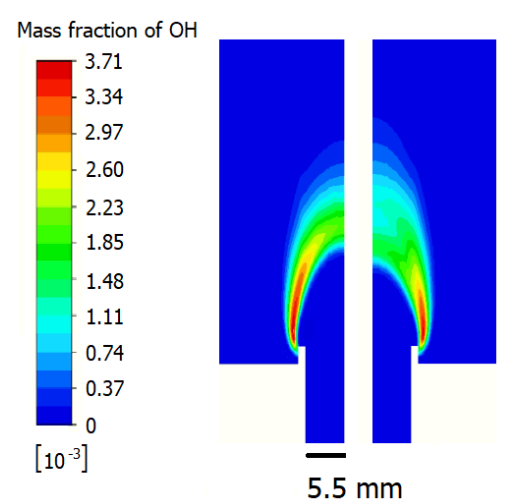

(b) Mass fraction of $\mathrm{OH}$.

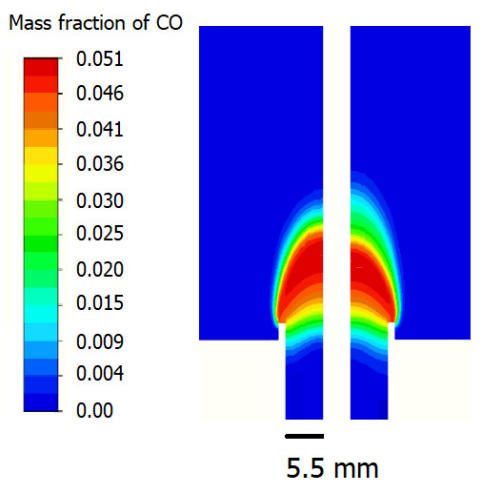

(c) Mass fraction of CO.

Figure 8: Comparison between reactive flow results obtained through CFD (left) and ROM (right) for the validation case 1.

\section{CONCLUDING REMARKS}

In this section, the conclusion of the laminar diffusion flame modelling with CFD and ROM are presented.

- Laminar diffusion flames stabilized in the Gülder burner were modeled using the Fluent software. Isothermal and reactive cases allowed the analysis of several properties of the flame, characterizing the flame and enabling the validation of the model;

- The use of the skeletal kinetics models in the CFD simulation enabled an analysis of several chemical species, thus underscoring the detailed flame structure;

- The analysis of the number of modes on the accuracy of the models, showed that by increasing the number of modes its possible to decrease the ROM error;

- The reduced order models for the isothermal case yielded similar results to those calculated by Fluent, with a maximum absolute error for the velocity of $0.0025 \mathrm{~m} / \mathrm{s}$ and for the mass fraction of $\mathrm{CH}_{4}$ of 0.0737 . 
- The reduced order models for the reactive case yielded similar results to those calculated by Fluent, for the velocity and temperature, with a maximum absolute error of $0.0019 \mathrm{~m} / \mathrm{s}$ and $98.9 \mathrm{~K}$, respectively. However, for the species mass fraction, the ROM results failed to describe the flame front details and the length of the highest concentration of $\mathrm{CO}$, despite the small maximum absolute error associated with the model.

- Future work will focus on understanding the error in the flame front description, analyzing the ROM methodology for the species.

\section{ACKNOWLEDGEMENTS}

Louise da C. Ramos developed this project while receiving funding from the European Union's

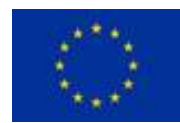
Horizon 2020 research and innovation programme under grant Agreement nr 766264. This work was performed while L.F. Figueira da Silva was on leave from the Institut Prime (CNRS, France). The authors also gratefully acknowledge the support provided by Conselho Nacional de Desenvolvimento Científico e Tecnológico, CNPq, under the Research Grants No. 403904/2016-1 and 110400/2019-4. This work was financed in part by the Coordenação de Aperfeiçoamento de Pessoal de Nível Superior - Brasil (CAPES) - Finance Code 001.

\section{REFERENCES}

ANSYS, 2009. “Gradient adaption approach”. $\quad$ ANSYS. $10 \quad$ Mar 2020 $<$ https://www.afs.enea.it/project/neptunius/docs/fluent/html/th/node395.htm>.

Asgari, S., Xaio, H. and Kaushik, S., 2017. "Applications of SVD ROM to thermal modeling”. In NAFEMS World Congress. Stockholm, Sweden, pp. 11-14.

Ben Salem, M., Roustant, O., Gamboa, F. and Tomaso, L., 2017. "Universal prediction distribution for surrogate models". SIAM/ASA Journal on Uncertainty Quantification, Vol. 5, pp. 1086-1109. doi:https://doi.org/10.1137/15M1053529.

Bourdrel, T., Bind, M.A., Béjot, Y., Morel, O. and Argacha, J.F., 2017. "Cardiovascular effects of air pollution”. Archives of Cardiovascular Disease, Vol. 110, pp. 634-642. doi:https://doi.org/10.1016/j.acvd.2017.05.003.

Celis, C. and Figueira da Silva, L.F., 2015. "Study of mass consistency LES/FDF techniques for chemically reacting flows". Combustion Theory and Modelling, Vol. 19, No. 4, pp. 465-494. doi: https://doi.org/10.1080/13647830.2015.1048828.

Cremer, M., Adams, B., Valentine, J., Letcavits, J.J. and Vierstra, S., 2002. "Use of CFD modeling to guide design and implementation of overfire air for nox control in coal-fired boilers". In 19th Annual International Pittsburgh Coal Conference. Pittsburgh, USA.

Cruz, J.J., Figueira da Silva, L.F., Escudero, F., Cepeda, F., Elicer-Cortés, J.C. and Fuentes, A., 2020. "Soot pyrometry by emission measurements at different wavelenghts in laminar axisymmetric flames". Combustion Science and Technology, Vol. (in press). doi:https://doi.org/10.1080/00102202.2020.1825401.

Da Costa Ramos, L., Di Meglio, F., Figueira da Silva, L.F. and Morgenthaler, V., 2020. "Reduced order model of laminar premixed inverted conical flames". In AIAA SciTech Forum. Orlando, USA. doi:https://doi.org/10.2514/6.2020-0416.

De Castro, R.R. and Figueira da Silva, L.F., 2019. "Experimental study of soot volume fraction and temperatue of laminar non-premixed ethylene-air flames”. In 25th ABCM International Congress of Mechanical Engineering. Minas Gerais, Brazil.

Escudero, F., Fuentes, A., Consalvi, J.L., Liu, F. and Demarco, R., 2016. "Unified behavior of soot production and radiative heat transfer in ethylene, propane and butane axisymmetric laminar diffusion flames at different oxygen indices". Fuel, Vol. 183, pp. 668 - 679. doi:https://doi.org/10.1016/j.fuel.2016.06.126.

Jerez, A., Cruz Villanueva, J.J., Figueira da Silva, L.F., Demarco, R. and Fuentes, A., 2019. "Measurements and modeling of PAH soot precursors in coflow ethylene/air laminar diffusion flames". Fuel, Vol. 236, pp. 452-460. doi: https://doi.org/10.1016/j.fuel.2018.09.047.

Kazakov, A. and Frenklach, M., 1984. "Reduced reaction sets based on gri-mech 1.2". The Combustion Laboratory at the University of California, Berkeley. 20 Nov. $2019<\mathrm{http} / / /$ combustion.berkeley.edu/drm/>.

LeCun, Y., Bengio, Y. and Hinton, G., 2015. "Deep learning”. Nature, Vol. 521, p. $436-444 . \quad$ doi: https://doi.org/10.1038/nature14539.

Luboz, V., Bailet, M., Grivot, C.B., Rochette, M., Diot, B., Bucki, M. and Payan, Y., 2018. "Personalized modeling for real-time pressure ulcer prevention in sitting posture". Journal of Tissue Viability, Vol. 27, pp. 54-58. doi: https://dx.doi.org/10.1016/j.jtv.2017.06.002.

McAllister, S., Chen, J. and Fernandez-Pello, A.C., 2011. Fundamentals of Combustion Processes. Springer, New York, USA, 1 st edition.

Orbegoso, E.M., Figueira da Silva, L.F. and Serfaty, R., 2016. "Comparative study of thermal radiation properties models in turbulent non-premixed sooting combustion". Numerical Heat Transfer, Part A: Applications, Vol. 69, No. 2, pp. 166-179. doi:https://doi.org/10.1080/10407782.2015.1052318. 
Turino, G.M., 1981. "Effect of carbon monoxide on the cardiorespiratory system: carbon monoxide toxicity, physiology and biochemistry". Circulation, Vol. 63, p. 253A-259A.

Viana, F.A.C., Haftka, R.T. and Steffen, V., 2017. "Optimization investigation on configuration parameters of spiralwound heat exchanger using genetic aggregation response surface and multi-objective genetic algorithm". Applied Thermal Engineering, Vol. 119, pp. 603-609. doi:https://doi.org/10.1016/j.applthermaleng.2017.03.100.

Wang, H., 2011. "Formation of nascent soot and other condensed-phase materials in flames". Proceedings of the Combustion Institute, Vol. 33, No. 1, pp. 41 - 67. doi:https://doi.org/10.1016/j.proci.2010.09.009.

Xiao, X., Fang, F., Buchan, A.G., Pain, C.C., Navon, I.M. and Muggeridge, A., 2015. "Non-intrusive reduced order modelling of the navier-stokes equations". Computer Methods in Applied Mechanics and Engineering, Vol. 293, pp. 522-541. doi:https://dx.doi.org/10.1016/j.cma.2015.05.015.

Zhang, A.Q., Thomson, M.J., Guo, H., Liu, F. and Smallwood, G.J., 2007. "Development of a parallel CFD code for modeling steady laminar axisymetric non-premixed co-flow flames with soot". In 2007 Spring Technical Meeting Combustion Institute. Canada, pp. 1-6.

Zhang, Y., Zhou, H., Xie, M., Fang, Q. and Wei, Y., 2010. "Modeling of soot formation in gas burner using reduced chemical kinetics coupled with CFD code". Chinese Journal of Chemical Engineering, Vol. 18, No. 6, pp. 967 - 978. doi:https://doi.org/10.1016/S1004-9541(09)60155-5.

\section{RESPONSIBILITY NOTICE}

The authors, Nicole Lopes Junqueira; Luís Fernando Figueira da Silva and Louise da Costa Ramos, are solely responsible for the printed material included in this paper. 